(CASE REPORT)

\title{
Metastasis of greater omentum
}

\author{
T Ramesh Rao and Suresh R Rao * \\ Department of Preclinical Sciences, Faculty of Medical Sciences, University of West Indies, St. Augustine Trinidad and \\ Tobago.
}

Publication history: Received on 30 June 2020; revised on 08 July 2020; accepted on 09 July 2020

Article DOI: https://doi.org/10.30574/wjarr.2020.7.1.0232

\begin{abstract}
Awareness of the variations and anomalous existence of some structures serve as useful guide for both in studies of human anatomy and in clinical practice today. The greater omentum characterizes an imperative intra-peritoneal structure with distinctive anatomic and pathologic features. It is primarily composed of fat, also contains blood vessels, nerves lymphatics and cellular tissues of the immune system. Its defensive role is related to its ability to phagocytize inflammatory process, contain bowel wall injuries, and become intricate in metastatic diseases of the peritoneal cavity. In our routine dissections with the purpose of preparation of the teaching and museum specimens, it was observed that in one of the elderly female cadaver, large pancake like mass was found covering the major portion of abdominal cavity, extending from greater curvature of stomach into the pelvis. Understanding the embryologic development, anatomic relationships, and pathologic processes of the greater omentum is critical to its complete and proper evaluation.
\end{abstract}

Keywords: Greater Omentum; Pathologic features; Variations

\section{Introduction}

The greater omentum is composed of a double layer of peritoneum that extends from the greater curvature of the stomach inferiorly. After a distance that usually ranges from 14 to $36 \mathrm{~cm}$, the greater omentum turns superiorly on itself to drape over the transverse colon and extend to the retroperitoneal pancreas. The greater omentum becomes adherent to but separate from the transverse mesocolon. The descending and ascending portions usually fuse to form a four-layer vascular fatty apron, with a space continuous with the lesser sac sometimes separating the two sets of layers. To its left, the greater omentum is continuous with the gastrosplenic ligament. Its right margin extends to the proximal duodenum.

Functionally the greater omentum acts as a store-house of fat and wraps an inflamed organ and prevents the spread of infection into the general peritoneal cavity. Developmentally the greater omentum is derived from the folding of the dorsal mesogastrium during the rotation of the stomach.

\section{Case report}

During routine gross anatomy dissection of the abdomen, for the purpose of teaching medical students, in Melaka Manipal Medical College (Manipal Campus), Manipal University, in a middle-aged female cadaver, we observed a large mass covering the major portion of abdominal cavity, extending from greater curvature of stomach into the pelvis.

The abdominal mass was thick, hard and somewhat nodular in the region of greater omentum, partially adherent to the intestine. We could observe nodular metastasis of almost all the organs of the abdominal cavity (Figure 1).

\footnotetext{
* Corresponding author: Suresh R Rao
} 


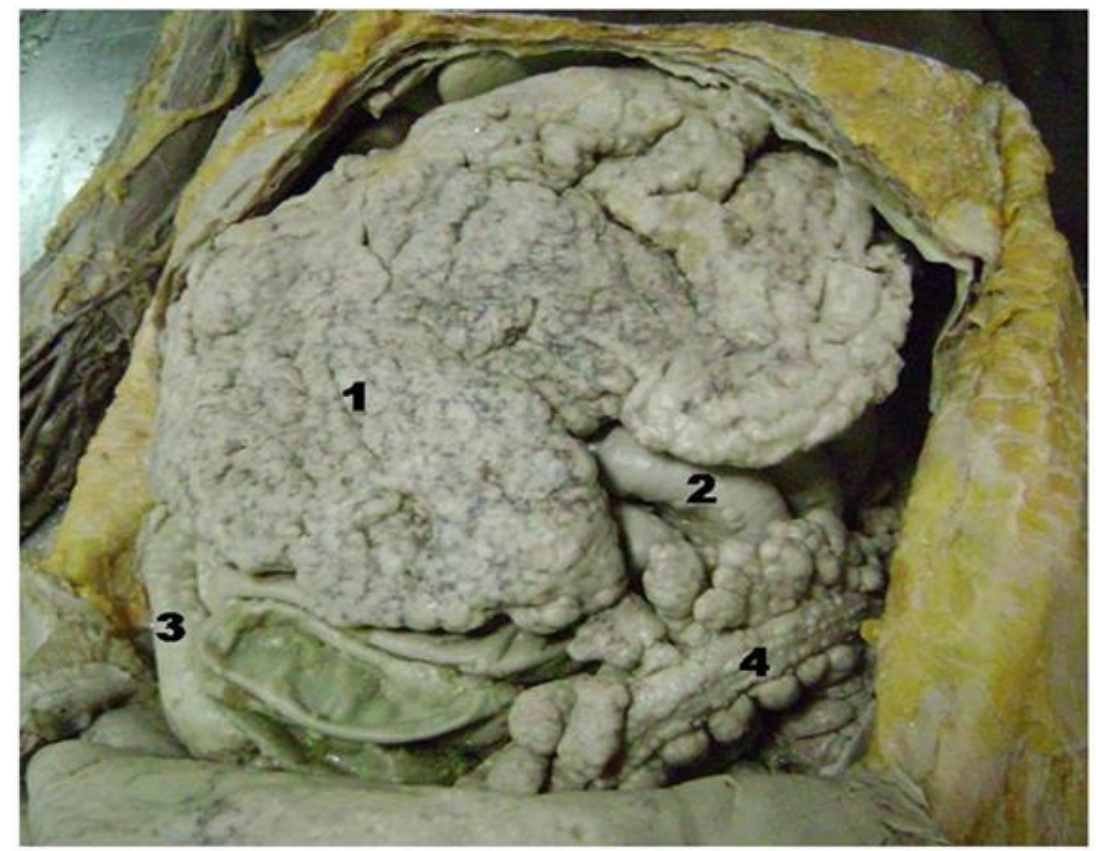

Figure 1 Metastasis of omentum. 1-Metastasis of greater omentum, 2- Metastasis of small intestine, 3- Metastasis of ascending colon, 4- Metastasis of sigmoid colon

Microscopic study of the omental mass also reveals the metastatic growth. Histopathological study of omental mass showed tumour composed of nests \& islands of malignant cells infiltrating the connective tissue \& stroma (Figure 2). The medical history of this middle aged female cadaver was not available. Following the dissection, the omental hard mass was photographed.

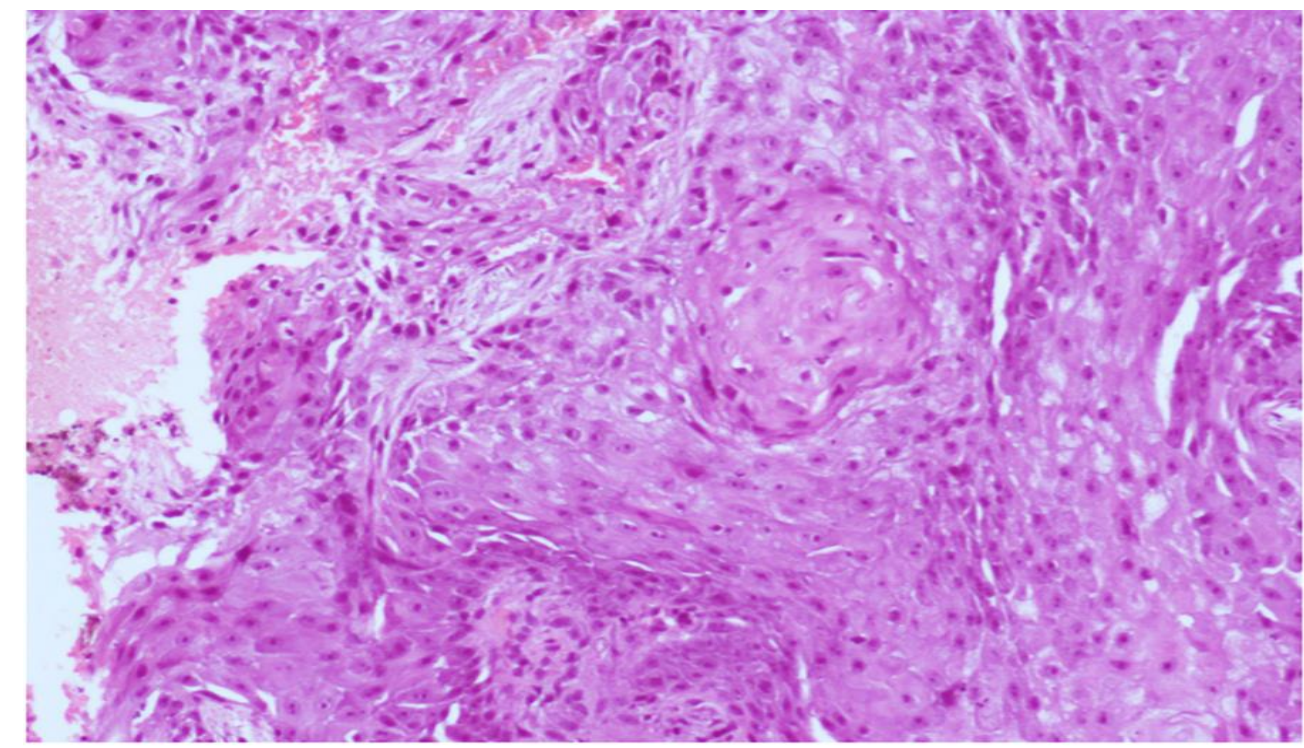

Figure 2 Histopathology of omental mass showing metastasis

\section{Discussion}

The significance of the greater omentum has been discovered recently by surgeons of various disciplines because it provides an excellent plastic material against inflammation and irradiation and for repair of defects that can be applied in the abdominal cavity; or it can be exteriorized and lengthened at a vascular pedicle and detached using microvascular anastomoses. Anatomic features, such as the volume of the omentum and the arrangement of the blood vessels, determine the lines of dissection. The eminent omental potential and the different biochemical and immunologic 
functions are unique, and can be related to specific anatomic structures, some of which may be drawn back to its embryologic sources. The ability of absorption and adhesion formation, neovascularization, and infection defense by the omentum protects against irradiation damage, accelerates healing of dead space, and improves the complication rate and quality of life after application to a wound bed [1].

Primary neoplasms of the omentum are rare and most often arise from mesodermal elements within the vessels and nerves that course through the omentum. When neoplasms occur, they are usually malignant. Metastatic disease involving the greater omentum is far more common than primary tumors. Although any tumor may secondarily involve the omentum, the most frequent malignant lesions that metastasize to the omentum include ovarian carcinoma and tumors of the colon and pancreas. Metastases from the stomach, appendix, kidney, uterus, and biliary tract may also metastasize to the greater omentum. Primary and secondary lymphoma of the omentum is rare [2].

Primary tumors of the greater omentum are made up of very diverse components pathologically, including leiomyosarcoma, fibrosarcoma, hemangiopericytoma, spindle-cell sarcoma, liposarcoma, lipoma, desmoid tumor, fibroma, and mesothelioma. To the best of our knowledge, only 22 cases of primary leiomyosarcoma of the greater omentum have ever been reported. Approximately $78 \%$ of patients with abdominal leiomyosarcoma have liver metastases, and the presence of unresectable metastatic liver tumors is the most important determinant of prognosis. Leiomyosarcoma originating in the greater omentum is rare, and most patients die of liver metastasis or peritoneal dissemination. The first choice of treatment for liver metastasis is surgery and the second is chemotherapy, while microwave coagulation therapy (MCT) has also been shown to be effective against hepatic tumors [3].

Ultrasound allows the detection and characterization primary tumours of the greater omentum, but determination of their precise anatomical location is usually difficult by Ultrasound. Computed tomography determines the omental origin of the tumour. Thus, when Ultrasound reveals an abdominal tumour of unknown origin, the possibility of an omental tumour, although rare, must be kept in mind and Computed Tomography should be performed [4].

In one of the case a large mass covering the whole intestine occupying the major portion of abdominal cavity, extending from greater curvature of stomach into the pelvis was noted, due to the advanced ovarian cancer, the ovaries were bulky, nodular \& hard. The abdominal mass was thick, hard and somewhat nodular in the region of greater omentum, partially adherent to the intestine. It was found that liver also involved by metastasis. Microscopic study of the omental mass also reveals the metastatic growth. Histopathological study of omental mass shows tumour composed of nests \& islands of malignant cells infiltrating the connective tissue \& stroma. The cells possess large pleomorphic hyperchromatic nuclei \&, inconspicuous nucleoli. Frequent mitotic activity \& mild to moderate inflammatory infiltrate is noted. Primary human omental adipocytes promote homing; migration and invasion of ovarian cancer cells, and adipokines including interleukin-8 (IL-8) mediate these activities [5].

\section{Conclusion}

Primary neoplasms of the omentum are rare, when neoplasms occur, they are usually malignant. Metastatic disease involving the greater omentum is far more common than primary tumors. The most frequent malignant lesions that metastasize to the omentum include mainly the ovarian carcinoma. Metastases from the colon, pancreas, stomach, appendix, kidney, uterus, and biliary tract may also metastasize to the greater omentum. Ovarian cancer is caused by changes in cancer-related genes. Some biological factors, such as genetic abnormalities, DNA ploidy, steroid receptors, affect the natural history of ovarian cancer. Surgical management like, cytodeductive surgery \& exploratory laparotomy is the first line treatment in all ovarian cancers patients followed by chemotherapy. Further validation using larger data sets warranted to prove the molecular and the biological properties of such carcinomas.

\section{Compliance with ethical standards}

\section{Acknowledgments}

Authors are thankful for the UWI and Subbaiah Institute of Medical Sciences for their continuous support in our research activities.

\section{Disclosure of conflict of interest}

Authors do not have any conflict of interest in the manuscript. 


\section{References}

[1] Liebermann-Meffert D. (2000). The greater omentum. Anatomy, embryology, and surgical applications. Surg Clin North Am, 80(1), 275-93.

[2] SusanWS, Robert EM, Paul MS and Raymond S. (1997). The Greater Omentum. AJR, 168, 683-687.

[3] Tadashi K, Tetsuya K, Yoshihiro K, Keiji K, Yoshihiko M, Tomohiro Y, Koji I and Keizo S. (2002). Leiomyosarcoma of the Greater Omentum with Liver Metastasis: Report of a Case. Surg Today, 32, 434-438.

[4] Ishida H and Ishida J. (1998). Primary tumours of the greater omentum. Eur Radiol. 8(9), 1598-601.

[5] Prashant AB and Karan BK. (2014). Greater Omental Pancake Tumour due to Metastasis of Ovarian Cancer - A Cadaveric Study. Journal of Clinical and Diagnostic Research, 8(1), 142-143.

\section{How to cite this article}

T Ramesh Rao and Suresh R Rao. (2020). Metastasis of Greater Omentum - case report. World Journal of Advanced Research and Reviews, 7(1), 183-186. 